\title{
WEYL-PARALLEL FORMS, CONFORMAL PRODUCTS AND EINSTEIN-WEYL MANIFOLDS*
}

\author{
FLORIN BELGUN ${ }^{\dagger}$ AND ANDREI MOROIANU ${ }^{\ddagger}$
}

\begin{abstract}
Motivated by the study of Weyl structures on conformal manifolds admitting parallel weightless forms, we define the notion of conformal product of conformal structures and study its basic properties. We obtain a classification of Weyl manifolds carrying parallel forms, and we use it to investigate the holonomy of the adapted Weyl connection on conformal products. As an application we describe a new class of Einstein-Weyl manifolds of dimension 4.
\end{abstract} omy.

Key words. Conformal products, conformally parallel forms, Weyl structures, reducible holon-

AMS subject classifications. Primary 53A30, 53C05, 53C29.

1. Introduction. A conformal structure on a smooth manifold $M$ is an equivalence class $c$ of Riemannian metrics modulo conformal rescalings, or, equivalently, a positive definite symmetric bilinear tensor with values in the square of the weight bundle $L$ of $M$. In contrast to the Riemannian situation, there is no canonical connection on a conformal manifold. Instead of the Levi-Civita connection, one can nevertheless consider the affine space of torsion-free connections preserving the conformal structure, called Weyl structures.

The fundamental theorem of conformal geometry states that this space is in oneto-one correspondence with the space of connections on the weight bundle $L$, and is thus modeled on the vector space of smooth 1-forms. It is worth noting that not every Weyl structure is (locally) the Levi-Civita connection of a Riemannian metric in the conformal class. This actually happens if and only if the corresponding connection on $L$ has vanishing curvature, in which case the Weyl structure is called closed. Every conformal problem involving closed Weyl structures is locally of Riemannian nature, so we will be mainly concerned with the case of non-closed Weyl structures.

Spin conformal manifolds with Weyl structures $D$ carrying parallel spinors have been studied in [10]. The basic idea, which allows the reduction of the problem to the Riemannian case, is that the curvature tensor of a non-closed Weyl structure is no longer symmetric by pairs. This fact eventually shows that the spin holonomy representation of a non-closed Weyl structure has no fixed points, except in dimension 4 , where genuine local examples do actually exist.

We consider here the analogous question for exterior forms: Characterize (locally) those conformal manifolds $(M, c)$ which carry an exterior form $\omega$ parallel with respect to some Weyl structure $D$. If $D$ is closed, it is (locally) the Levi-Civita connection of some metric $g \in c$ and $D$-parallel forms correspond to fixed points of the Riemannian holonomy representation on the exterior bundle. By the de Rham theorem, and the fact that the space of fixed points of a tensor product representation is just the tensor product of the corresponding spaces of each factor, one may assume that the holonomy

${ }^{*}$ Received September 1, 2010; accepted for publication December 14, 2010. This work was partially supported by the French-German cooperation project Procope no. 17825PG. The first named author was equally supported by the Schwerpunktprogramm 1154 Globale Differentialgeometrie of the DFG.

${ }^{\dagger}$ Fachbereich Mathematik, Universität Hamburg, Bundesstr. 55, D-20146 Hamburg, Germany (florin.belgun@math.uni-hamburg.de).

$\ddagger$ CMLS, École Polytechnique, UMR 7640 du CNRS, 91128 Palaiseau, France (am@math.poly technique.fr). 
acts irreducibly on $T M$. In this case, the Berger-Simons theorem provides the list of possible holonomy groups, so the problem reduces to an algebraic (although far from being trivial) computation.

Back to the conformal setting, we remark that we can restrict ourselves to the case of weightless forms since otherwise the Weyl structure would be automatically closed. By choosing a Riemannian metric $g \in c$, the equation $D \omega=0$ becomes

$$
\left.\left.\nabla_{X}^{g} \omega=\theta \wedge X\right\lrcorner \omega-X^{b} \wedge \theta^{\sharp}\right\lrcorner \omega \quad \forall X \in T M,
$$

where $\nabla^{g}$ is the Levi-Civita covariant derivative of $g$ and $\theta$ is the connection form of $D$ in the trivialization of $L$ determined by $g$. Exterior forms satisfying (1) are called locally conformal parallel forms in [5] and are shown to define, under some further conditions, harmonic sections of the corresponding sphere bundles.

We start by remarking that a nowhere vanishing exterior $p$-form $\omega(0<p<$ $\operatorname{dim}(M))$ can not be parallel with respect to more than one Weyl structure. In fact $\omega$ defines a unique "minimal" Weyl structure $D^{\omega}$ which is the only possible candidate for having $D \omega=0$. We next apply the Merkulov-Schwachhöfer classification of torsionfree connections with irreducible holonomy [9] to the Weyl structure $D^{\omega}$. A quick analysis of their tables shows that the possible (non-generic) holonomy groups of irreducible Weyl structures are all compact (except in dimension 4, where the solutions to our problem turn out to correspond to Hermitian structures - see Lemma 5.7). But, of course, a Weyl structure with compact (reduced) holonomy is closed since its holonomy bundle defines (local) Riemannian metrics which are tautologically $D$ parallel.

It remains to study the reducible case, which, unlike in the Riemannian situation, is more involved. First of all, we extend the de Rham theorem to the conformal setting. To do this, we need to define the notion of conformal products. Indeed, in contrast to Riemannian geometry, there is no canonical conformal structure on a product $M_{1} \times M_{2}$ of two conformal manifolds $\left(M_{1}^{n_{1}}, c_{1}\right)$ and $\left(M_{2}^{n_{2}}, c_{2}\right)$ induced by the two conformal structures alone. The algebraic reason is, of course, that the group $\mathrm{CO}\left(n_{1}\right) \times \mathrm{CO}\left(n_{2}\right) \subset \mathrm{GL}\left(n_{1}+n_{2}, \mathbb{R}\right)$ is not included in $\mathrm{CO}\left(n_{1}+n_{2}\right)$.

On the other hand, a property characterizing the Riemannian product $(M, g)$ of two Riemannian manifolds $\left(M_{1}, g_{1}\right)$ and $\left(M_{2}, g_{2}\right)$ is the existence of two complementary orthogonal Riemannian submersions $p_{i}:(M, g) \rightarrow\left(M_{i}, g_{i}\right)$ (here, complementary means that $T M$ is the direct sum of the kernels of $d p_{i}$ and orthogonal means that these kernels are orthogonal at each point). Generalizing this to conformal geometry, a conformal structure on the manifold $M:=M_{1} \times M_{2}$ is said to be a conformal product of $\left(M_{1}, c_{1}\right)$ and $\left(M_{2}, c_{2}\right)$ if the canonical submersions $p_{1}: M \rightarrow M_{1}$ and $p_{2}: M \rightarrow M_{2}$ are orthogonal conformal submersions.

In Section 4 we show that every conformal product carries a unique adapted reducible Weyl structure $D$ preserving the two factors, and conversely, every reducible Weyl structure induces a local conformal product structure.

The similarities with the Riemannian case stop here, however, since the factors of a conformal product do not carry canonical Weyl structures (in fact the restrictions of the adapted Weyl structure $D$ to each slice $\left\{x_{1}\right\} \times M_{2}$ or $M_{1} \times\left\{x_{2}\right\}$ of the conformal product $M_{1} \times M_{2}$ depends on $x_{1}$ and $x_{2}$ ), so it is not possible to interpret the space of $D$-parallel forms on $M_{1} \times M_{2}$ in terms of the two factors.

On the other hand, the lack of symmetry of the curvature tensor of $D$ mentioned above, allows us to show (in Section 5) that every parallel form on a conformal product with non-closed adapted Weyl structure is of pure type and eventually has to be the 
weightless volume form of one of the factors, exception made of the 2-dimensional conformal products and of some conformal products of dimension 4 (which are described in detail in Section 6).

As an application, we present an explicit construction of new families of EinsteinWeyl structures in dimension 4, using conformal products of surfaces by means of solutions of a non-linear equation of Toda type, among which a special class consists of bi-harmonic functions. These reduce to the well-known examples of hyper-Hermitian surfaces constructed by Joyce [7] in the particular case where the bi-harmonic function is the real part of a holomorphic function on $\mathbb{C}^{2}$, examples that fully cover the cases of conformal multi-products in dimension larger than 2, i.e., of a Weyl structure leaving invariant more than one pair of orthogonal proper subspaces.

Note that the conformal product Ansatz already occurred (although without being named) in the study of 3-dimensional Einstein-Weyl structures [4]. The Einstein-Weyl structure on this conformal product is, however, different from the adapted one.

A similar question about holonomy on $n$-dimensional conformal manifolds $(M, c)$ was studied by S. Armstrong in [1]. He considers the holonomy of the canonical Cartan connection, which is not an affine connection on $T M$ but a (uniquely defined) linear connection on an $n+2$-rank vector bundle of Lorentzian signature, and classifies the occurring holonomy groups. A decomposition theorem is also given in this context, if the Cartan connection leaves a $k$-dimensional subspace invariant, for $2 \leq k \leq n$ : Summarized in the terms of our present paper, $(M, c)$ turns out to be, in this case, a closed conformal product with Einstein factors (plus some relation between the scalar curvatures).

We see therefore that despite the obvious geometric particularity of a general conformal product structure, no restriction on the holonomy of the Cartan connection (also called conformal holonomy) is implied. This fact brings us to the first of the following open questions about conformal products:

1. Is there any invariant characterization of the underlying conformal structure of a conformal product?

2. Can the multiple conformal products (i.e., admitting more than one pair of orthogonal conformal submersions) be characterized geometrically?

3. Which are the possible reduced holonomies of a Weyl structure?

On the other hand, as the results of [4] and Section 6 suggest, the conformal product Ansatz is expected to have further applications.

2. Preliminaries. Let $M^{n}$ be a manifold and let $P$ denote the principal bundle of frames. The weight bundle of $M$ is the real line bundle $L$ associated to $P$ via the representation $|\operatorname{det}|^{1 / n}$ of $\mathrm{GL}(n, \mathbb{R})$. More generally one can define the $k$-weight bundle $L^{k}$ for every $k \in \mathbb{R}$, associated to $P$ via the representation $|\operatorname{det}|^{k / n}$. Obviously $L^{k} \otimes L^{p} \cong L^{k+p}$ and $L^{-n} \cong\left|\Lambda^{n}\left(T^{*} M\right)\right|=\delta M$, which is the bundle of densities on $M$, a trivial line bundle (associated to $P$ via the representation $|\operatorname{det}|^{-1}$ ) even if $M$ is not orientable. Positive densities are geometrically meaningful as "absolute values" of volume forms and positive global densities induce Lebesgue-like measures on $M$ (like the well-known Riemannian volume element of a Riemannian manifold).

As the weight bundles are powers of $\delta M$, the notion of positivity is still welldefined; more precisely, a section of $L^{k}$ is positive if it takes values in $P \times_{|\operatorname{det}| k / n} \mathbb{R}^{+} \subset$ $L^{k}$. A weighted tensor on $M$ is a section of $T M^{\otimes a} \otimes T^{*} M^{\otimes b} \otimes L^{k}$ for some $a, b \in \mathbb{N}$ and $k \in \mathbb{R}$. Its weight is by definition the real number $a-b+k$.

Definition 2.1. A conformal structure on $M$ is a symmetric positive definite 
bilinear form c on $T M \otimes L^{-1}$, or, equivalently, a symmetric positive definite bilinear form on $T M$ with values in $L^{2}$.

A conformal structure on $M$ can also be seen as a reduction $P\left(\mathrm{CO}_{n}\right)$ of $P$ to the conformal group $\mathrm{CO}_{n} \cong \mathbb{R}^{+} \times \mathrm{O}_{n} \subset \mathrm{GL}(n, \mathbb{R})$.

We denote by $\Lambda_{0}^{k} M:=\Lambda^{k} M \otimes L^{k}$ the bundle of weightless exterior forms of degree $k$. The conformal structure defines an isomorphism between weightless vectors and 1-forms: $T M \otimes L^{-1} \cong \Lambda_{0}^{1} M$. The scalar product $c$ on $\Lambda^{1} M \otimes L$ induces a scalar product, also denoted by $c$, on the bundles $\Lambda_{0}^{k} M$. Moreover, the exterior product maps $\Lambda_{0}^{k} M \otimes \Lambda_{0}^{n-k} M$ onto $\Lambda_{0}^{n} M \cong \mathbb{R}$, thus defining the Hodge operator $*: \Lambda_{0}^{k} M \rightarrow \Lambda_{0}^{n-k} M$ by

$$
\omega \wedge \sigma=c(\omega, \sigma), \quad \forall \omega, \sigma \in \Lambda_{0}^{k} M
$$

There is a one-to-one correspondence between positive sections $l$ of $L$ and Riemannian metrics on $M$, given by the formula

$$
c(X, Y)=g(X, Y) l^{2}, \quad \forall X, Y \in T M .
$$

Definition 2.2. A Weyl structure on a conformal manifold is a torsion-free connection on $P\left(\mathrm{CO}_{n}\right)$.

By (2), the Hodge operator is parallel with respect to every Weyl structure.

Theorem 2.3. (Fundamental theorem of Weyl geometry) There is a one-to-one correspondence between Weyl structures and covariant derivatives on $L$.

Proof. Every connection on $P$ induces a covariant derivative $D$ on $T M$ and on $L$. The connection is a Weyl structure if and only if $D$ satisfies $D_{X} Y-D_{Y} X=[X, Y]$ and $D_{X} c=0$ for all vector fields $X, Y$ on $M$. Like in the Riemannian situation, these two relations are equivalent to the Koszul formula

$$
\begin{aligned}
2 c\left(D_{X} Y, Z\right)= & D_{X}(c(Y, Z))+D_{Y}(c(X, Z))-D_{Z}(c(X, Y)) \\
& +c([X, Y], Z)+c([Z, X], Y)+c([Z, Y], X),
\end{aligned}
$$

for all vector fields $X, Y, Z$ on $M$. We thus see that every covariant derivative $D$ on $L$ induces by the formula above a covariant derivative on $T M$, and thus on $P$, which is clearly torsion-free and satisfies $D c=0$.

A Weyl structure $D$ is called closed (resp. exact) if $L$ carries a local (resp. global) $D$-parallel section. As Riemannian metrics in the conformal class $c$ correspond to positive sections of $L$, it follows immediately that $D$ is closed (resp. exact) if and only if $D$ is locally (resp. globally) the Levi-Civita connection of a metric $g \in c$.

If $D$ and $D^{\prime}$ are covariant derivatives on $L$, their difference is determined by a 1-form $\tau: D_{X} l-D_{X}^{\prime} l=\tau(X) l$ for all $X \in T M$ and sections $l$ of $L$. From (4) we easily obtain

$$
D_{X} Y-D_{X}^{\prime} Y=\tau(X) Y+\tau(Y) X-c(X, Y) \tau
$$

for all vector fields $X, Y$. Here we note that the last term on the right hand side, which is a section of $L^{2} \otimes \Lambda^{1} M$, is identified with a vector field using the conformal structure. 
For every $X \in T M$ we define the endomorphism $\tilde{\tau}_{X}$ on $T M$ and on $L$ by

$$
\tilde{\tau}_{X}(Y):=\tau(X) Y+\tau(Y) X-c(X, Y) \tau, \quad \tilde{\tau}_{X}(l):=\tau(X) l,
$$

and extend it as a derivation to all weighted tensor bundles (in particular $\tilde{\tau}_{X}$ is the scalar multiplication by $k \tau(X)$ on $\left.L^{k}\right)$. We then have

$$
D_{X}-D_{X}^{\prime}=\tilde{\tau}_{X}
$$

on all weighted bundles.

Consider now a metric $g$ in the conformal class $c$, or equivalently, a positive section $l$ of $L$ trivializing $L$. Let $D$ be a Weyl structure on $(M, c)$ and let $\theta \in \Omega^{1}(M, \mathbb{R})$ be the connection form of $D$ on $L$ with respect to the gauge $l$ :

$$
D_{X} l=\theta(X) l, \quad \forall X \in T M .
$$

The 1-form $\theta$ is called the Lee form of $D$ with respect to $g$. The curvature of $D$ on $L$ is the two-form $F:=d \theta$ called the Faraday form.

Let $R^{D}$ denote the curvature tensor of a Weyl structure $D$, defined as usual for vector fields $X, Y$ and $Z$ by $R_{X, Y}^{D} Z=\left[D_{X}, D_{Y}\right] Z-D_{[X, Y]} Z$. We also view $R^{D}$ as a section of $T^{*} M^{\otimes 4} \otimes L^{2}$ by the formula $R^{D}(X, Y, Z, T)=c\left(R_{X, Y}^{D} Z, T\right)$. In contrast to the Riemannian case, $R^{D}$ is not symmetric by pairs, and a straightforward calculation shows that the symmetry failure is measured by the Faraday form $F$ of $D$ :

$$
\begin{aligned}
R^{D}(X, Y, Z, T)-R^{D}(Z, T, X, Y)= & (F(X) \wedge Y-F(Y) \wedge X)(Z, T) \\
& +F(X, Y) c(Z, T)-F(Z, T) c(X, Y),
\end{aligned}
$$

where, for a (weighted) endomorphism $A \in \operatorname{End}(T M) \otimes L^{k}$, and vectors $X, Y, Z, T$ :

$$
(A(X) \wedge Y)(Z, T):=c(A(X), Z) c(Y, T)-c(A(X), T) c(Y, Z) .
$$

3. Conformal submersions. If $\left(M^{m}, c\right)$ and $\left(N^{n}, c^{\prime}\right)$ are conformal manifolds, a conformal map is a smooth map $f: M \rightarrow N$ such that

$$
\left.d f\right|_{(\operatorname{ker} d f)^{\perp}}:(\operatorname{ker} d f)^{\perp} \rightarrow d f\left(T_{x} M\right) \subset T_{f(x)} N
$$

is a conformal isomorphism for every $x \in M$. A conformal map which is a submersion is called a conformal submersion.

Lemma 3.1. Let $\left(M^{m}, c\right)$ be a conformal manifold and let $p: M \rightarrow N$ be a submersion onto a manifold $N^{n}$. Then the pull-back of the weight bundle of $N$ is canonically isomorphic to the weight bundle of $M$.

Proof. Let $L^{\prime}$ and $L$ denote the weight bundles of $N$ and $M$ respectively. We decompose $T M=\operatorname{ker} d p \oplus(\operatorname{ker} d p)^{\perp}$ into the vertical and horizontal distributions. We will show that $p^{*}\left(L^{\prime}\right)^{-n}$ is canonically isomorphic to $L^{-n}$. Every element $\left(l^{\prime}\right)^{-n}$ of the fiber of $\left(L^{\prime}\right)^{-n} \simeq \delta N$ at $y \in N$ can be represented by the density $\left(l^{\prime}\right)^{-n}:=\left|\varepsilon_{1} \wedge \ldots \wedge \varepsilon_{n}\right|$ where $\left\{\varepsilon_{i}\right\}$ is some basis of $T^{*} N_{y}$. For every $x \in p^{-1}(y)$ we then associate to the element $p^{*}\left(l^{\prime}\right)_{y}^{-n}$ the conformal norm of $\left(p^{*} \varepsilon_{1}\right)_{x} \wedge \ldots \wedge\left(p^{*} \varepsilon_{n}\right)_{x}$, which is an element of $L_{x}^{-n}$. It is straightforward to check that this isomorphism does not depend on the choice of the basis.

REMARK 3.2. This result only holds for $n \geq 1$ since we need at least one nonvanishing 1-form in order to produce a weight on $N$. 
LEMMA 3.3. Let $p: M \rightarrow N$ be a submersion with connected fibers from a conformal manifold $\left(M^{m}, c\right)$ onto a manifold $N^{n}$. Assume that the horizontal distribution $H:=(\operatorname{ker} d f)^{\perp}$ is parallel with respect to some Weyl structure D. Then the pull-back to $M$ of every covariant weighted tensor on $N$ is D-parallel in the vertical directions.

Conversely, a covariant weighted tensor on $M$ which is horizontal and D-parallel in the vertical directions, is the pull-back of a covariant weighted tensor on $N$.

Proof. We first show that $D_{V}\left(p^{*} \omega\right)=0$ for all 1-forms $\omega$ on $N$. If $W$ is a vertical vector field, $D_{V} W$ is again vertical, so $0=p^{*} \omega\left(D_{V} W\right)=\left(D_{V}\left(p^{*} \omega\right)\right)(W)$. Next, if $X$ is another vector field on $N$ and $\tilde{X}$ denotes its horizontal lift, $p^{*} \omega(\tilde{X})$ is constant on each fiber of $p$, so $0=V \cdot\left(p^{*} \omega(\tilde{X})\right)=\left(D_{V}\left(p^{*} \omega\right)\right)(\tilde{X})+\left(p^{*} \omega\right)\left(D_{V} \tilde{X}\right)$. On the other hand $D_{V} \tilde{X}=D_{\tilde{X}} V+[V, \tilde{X}]$ vanishes (because $D_{\tilde{X}} V$ and $[V, \tilde{X}]$ are vertical, and $D_{V} \tilde{X}$ is horizontal), so finally $D_{V}\left(p^{*} \omega\right)=0$.

Lemma 3.1 shows that the pull-back of the weight bundle $L^{\prime}$ of $N$ is isomorphic to the weight bundle $L$ of $M$. Moreover, the calculation above shows that

$$
D_{V}\left(p^{*} l^{\prime}\right)=0
$$

for every section $l^{\prime}$ of $L^{\prime}$ and vertical vector field $V \in$ ker $d f$. Since the 1-forms and the sections of $L^{\prime}$ generate the whole algebra of covariant weighted tensors on $N$, this proves the first part of the lemma.

For the converse part, we first show that a section $l$ of $L$ which is parallel in vertical directions is the pull-back of a section of $L^{\prime} \rightarrow N$. Indeed, if we take any global nowhere vanishing section $l^{\prime}$ of $L^{\prime}$, one can write $l=f p^{*} l^{\prime}$ for some function $f$ which by (9) is constant in vertical directions, i.e. $f$ is the pull-back of some function $f^{\prime}$ on $N$, so finally $l=p^{*}\left(f^{\prime} l^{\prime}\right)$.

Let now $Q: T M^{\otimes k} \rightarrow L^{r}$ be a covariant weighted tensor field on $M$ such that $Q\left(X_{1}, \ldots, X_{k}\right)=0$ whenever one of the $X_{i}$ is vertical, and which is $D$-parallel in the vertical directions. For every $y \in N$ and $x \in p^{-1}(y) \subset M$ we define

$$
\bar{Q}_{y}\left(Y_{1}, \ldots, Y_{k}\right):=Q_{x}\left(\tilde{Y}_{1}, \ldots, \tilde{Y}_{k}\right)
$$

where $Y_{1}, \ldots, Y_{k} \in T_{y} N$ are arbitrary vectors, and $\tilde{Y}_{1}, \ldots, \tilde{Y}_{k} \in T_{x} M$ are their horizontal lifts. This definition makes sense thanks to the identification of $p^{*} L^{\prime} \simeq L$ of Lemma 3.1. In order to show that it is independent of the choice of $x \in p^{-1}(y)$, we need to check that the right hand side is a weight which is $D$-parallel in vertical directions. This follows from the relations $D_{V} \tilde{Y}_{i}=0$, proved above, and the hypothesis $D_{V} Q=0$.

COROLlary 3.4. Under the hypothesis of the previous lemma, there exists a unique conformal structure on $N$ turning $p$ into a conformal submersion.

Proof. Let $c_{1}$ denote the restriction of the conformal structure on $M$ to the horizontal distribution. Since the horizontal distribution is $D$-parallel, the same holds for $c_{1}$. Lemma 3.3 thus shows that $c_{1}$ is the pull-back of some weighted tensor $c^{\prime}$ on $N$, which is clearly a conformal structure on $N$.

REMARK 3.5. We can extend now the result of the Lemma 3.3 to any weighted tensor on $N$, respectively on $M$, because on a conformal manifold every tensor can be seen as a covariant one (with the appropriate weight). 
4. Conformal products. Let $\left(M_{1}, c_{1}\right)$ and $\left(M_{2}, c_{2}\right)$ be two conformal manifolds, $M=M_{1} \times M_{2}$ and let $p_{i}: M \rightarrow M_{i}$ be the canonical submersions.

Definition 4.1. A conformal structure on the manifold $M:=M_{1} \times M_{2}$ is said to be a conformal product of $\left(M_{1}, c_{1}\right)$ and $\left(M_{2}, c_{2}\right)$ if and only if the canonical submersions $p_{1}: M \rightarrow M_{1}$ and $p_{2}: M \rightarrow M_{2}$ are orthogonal conformal submersions.

For later use, we describe the construction of a conformal product structure in terms of weight bundles:

Proposition 4.2. Given two conformal manifolds $\left(M_{1}^{n_{1}}, c_{1}\right)$, resp. $\left(M_{2}^{n_{2}}, c_{2}\right)$, there is a one-to-one correspondence between the set of conformal product structures on $M:=M_{1} \times M_{2}$ and the set of pairs of bundle homomorphisms $P_{1}: L \rightarrow L_{1}$ and $P_{2}: L \rightarrow L_{2}$, whose restrictions to each fiber are isomorphisms, such that the following diagram is commutative (here $L, L_{1}, L_{2}$ denote the weight bundles of $M, M_{1}$, and $M_{2}$ respectively):

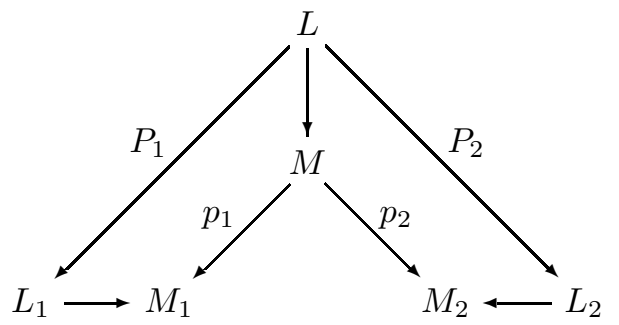

Proof. It is a general fact that for a conformal map $f:(M, c) \rightarrow\left(N, c^{\prime}\right)$ between two conformal manifolds, there is a canonically associated bundle map $f^{L}: L_{M} \rightarrow$ $L_{N}$ of the weight bundles, isomorphic on each fiber (take any non-zero vector $X$ in $(\text { ker } d f)^{\perp}$ and define $\left.f^{L}(\sqrt{c(X, X)})=\sqrt{c^{\prime}\left(f_{*} X, f_{*} X\right)}\right)$.

Therefore, given a conformal product structure on $M \simeq M_{1} \times M_{2}$ (i.e., a pair of conformal submersions $p_{1}: M \rightarrow M_{1}$, resp. $p_{2}: M \rightarrow M_{2}$ ), we associate to it the induced bundle homomorphisms $P_{i}:=p_{i}^{L}$, such that the diagram (11) commutes.

Conversely, let $M=M_{1} \times M_{2}$ and let $L$ denote the weight bundle of the product manifold $M$ (which does not have any conformal structure yet). Let $P_{i}: L \rightarrow L_{i}$ be line bundle homomorphisms making the diagram (11) commutative. The condition that $P_{i}$ are isomorphic on each fiber just means that the pull-back bundles $p_{i}^{*} L_{i}$ are both isomorphic with $L$. We then define $\tilde{c}_{i} \in \operatorname{Sym}^{2}(M) \otimes p_{i}^{*}\left(L_{i}^{2}\right) \cong \operatorname{Sym}^{2}(M) \otimes L^{2}$ by $\tilde{c}_{i}(X, Y):=p_{i}^{*}\left(c_{i}\left(\left(p_{i}\right)_{*}(X),\left(p_{i}\right)_{*}(Y)\right)\right)$ and $c=\tilde{c}_{1}+\tilde{c}_{2}$.

The following theorem, which is the main result of this section, establishes the existence of a unique adapted Weyl structure on a conformal product:

TheOREM 4.3. A conformal structure $c$ on a manifold $M$ is a (local) conformal product structure if and only if it carries a Weyl structure with reducible holonomy. Moreover, the correspondence between the conformal product structures and the Weyl structures with reduced holonomy is one-to-one.

Proof. Let us first prove that if a conformal manifold $(M, c)$ carries a Weyl structure $D$ with reduced holonomy, then it is locally a conformal product. Let $T M=$ $H_{1} \oplus H_{2}$ be a $D$-invariant splitting of the tangent bundle. Because $D$ is torsion-free, the distributions $H_{1}$ and $H_{2}$ are integrable, therefore we have two orthogonal foliations on $M$ tangent to these distributions. Locally, $M$ is then a product manifold $M_{1} \times M_{2}$, 
and the foliations above are the fibers of the canonical projections $p_{i}: M \rightarrow M_{i}$. Corollary 3.4 then shows that there exist conformal structures $c_{1}, c_{2}$ on $M_{1}$, resp. $M_{2}$, such that the canonical projections are conformal submersions.

Conversely, suppose $(M, c)$ is a conformal product with factors $\left(M_{1}, c_{1}\right)$, resp. $\left(M_{2}, c_{2}\right)$. We look for a Weyl structure $D$ that preserves the canonical splitting $T M=$ $H_{1} \oplus H_{2}$, with $H_{1}:=\operatorname{ker} d p_{2}$ and $H_{2}:=\operatorname{ker} d p_{1}$.

By Theorem 2.2, the set of Weyl structures is in 1-1 correspondence with the set of connections on the weight bundle. Therefore, it is enough to specify the corresponding connection $D$ on $L$. We describe $D$ using the diagram (11) as follows: the horizontal space $H_{l}$ of $D$ at $l \in L$ is the direct sum of $\operatorname{ker} d P_{1}$ and $\operatorname{ker} d P_{2}$. This horizontal space defines a linear connection because the maps $P_{1}, P_{2}$ commute with the scalar multiplication on the fibers. In terms of covariant derivative, this definition amounts to say that the pull-back of a section of $L_{1}$ (resp. $\left.L_{2}\right)$ is $D$-parallel in the direction of $H_{2}$ (resp. $H_{1}$ ). We need to show that the induced Weyl structure $D$ preserves $H_{1}$ and $\mathrm{H}_{2}$.

Since the rôles of $H_{1}$ and $H_{2}$ are symmetric, it is enough to prove that $c\left(D_{X} Y, Z\right)=0$ for all vector fields $X, Y \in H_{1}$ and $Z \in H_{2}$. Of course, we may assume that $X, Y$ are lifts of vector fields on $M_{1}$ and $Z$ is a lift of a vector field on $M_{2}$. Then the brackets $[X, Z]$ and $[Y, Z]$ vanish, because the vector fields $X$ and $Y$, resp. $Z$ are defined on different factors of the product $M_{1} \times M_{2}$, and the scalar products $g(X, Z)=g(Y, Z)=0$ for the same reason. The only a priori non-vanishing terms in the Koszul formula (4) are thus:

$$
2 c\left(D_{X} Y, Z\right)=-D_{Z}(c(X, Y))+c([X, Y], Z) \text {. }
$$

The first term vanishes by the definition of $D$ and the second one because $[X, Y] \in H_{1}$ and $Z \in H_{2}$.

Definition 4.4. The Weyl structure defined on a conformal product $(M, c)$ by the result above is called the adapted Weyl structure.

Definition 4.5. A conformal product $(M, c)$ is called a closed conformal product if the adapted Weyl structure is closed.

It is easy to check that a conformal product is closed if and only if the diagram (11) can be completed by bundle homomorphisms $Q_{i}: L_{i} \rightarrow L_{0}$, isomorphic on each fiber (where $L_{0}$ is the weight bundle of the point manifold $\bullet$ ), such that the resulting diagram is commutative as well:

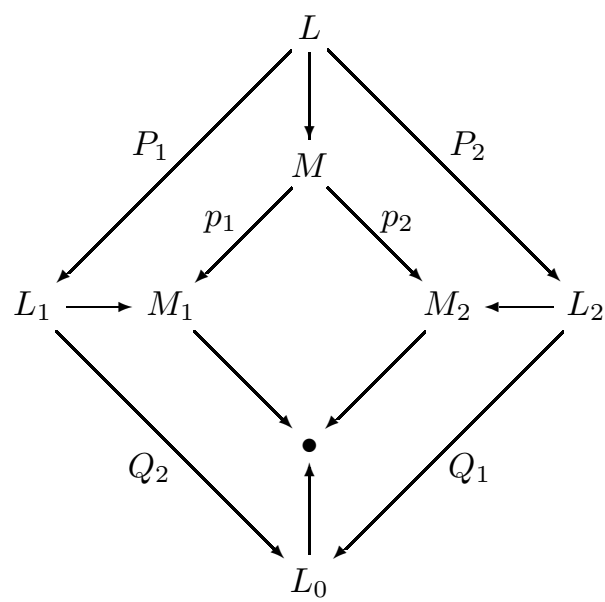


Lemma 4.6. Let $F$ be the Faraday form of the adapted Weyl structure on a conformal product. Then $F(X, Y)=0$ if $X, Y \in H_{1}$ or $X, Y \in H_{2}$.

Proof. Let $l_{1}$ be a section of the weight bundle $L_{1}$ of $M_{1}$. Lemma 3.1 shows that its pull-back can be identified with a section $l$ of $L$, and Lemma 3.3 shows that $D_{X} l=0$ for every $X \in H_{2}$. Let $X, Y$ be sections of $H_{2}$. Since $H_{2}$ is involutive, $[X, Y]$ is a section of $\mathrm{H}_{2}$, so

$$
F(X, Y) l=D_{X} D_{Y} l-D_{Y} D_{X} l-D_{[X, Y]} l=0 .
$$

The vanishing of $F$ on $H_{1}$ is similar.

REMARK 4.7. One can show that, for any given distribution $E$ on a conformal manifold $M$, there is a unique adapted Weyl structure $\nabla$, in the sense that some naturally defined tensors, depending on the splitting of $T M \simeq E \oplus E^{\perp}$ have minimal covariant derivative (see [3], Prop. 3.3).

LEMMA 4.8. Let $\left(M_{1}, c_{1}\right)$ and $\left(M_{2}, c_{2}\right)$ be two conformal manifolds and let $c$ be a conformal product structure on $M=M_{1} \times M_{2}$ with adapted Weyl structure $D$. Then each slice $M_{1} \times\{y\} \simeq M_{1}$ carries a Weyl structure $D^{y}$ such that $p_{1}^{*}\left(D_{X}^{y} T\right)=D_{X}\left(p_{1}^{*} T\right)$ at points of the form $(x, y)$ for all vectors $X$ and tensor fields $T$ on $M_{1}$. The Weyl structure $D$ is closed if and only if all connections $D^{y}$ coincide.

Proof. The restriction to each slice $M_{1} \times\{y\}$ defines a covariant derivative $D^{y}$ on $M_{1}$, which by Lemmas 3.1 and 3.3 preserves the conformal structure of $M_{1}$. In order to prove the last statement, it is enough to consider the case when $T$ is a vector field on $M_{1}$. We consider vector fields $U_{2}$ on $M_{2}$ and $X_{1}, Y_{1}, Z_{1}$ on $M_{1}$, and denote their canonical lifts to $M$ by $U$, respectively by $X, Y, Z$. Lemma 3.3 shows that $D_{U} Y=0$, which together with $[U, X]=0$ yields $R^{D}(U, X, Y, Z)=c\left(D_{U} D_{X} Y, Z\right)$. On the other hand, $R_{Y, Z}^{D} U$ is tangent to $M_{2}$, so $R^{D}(Y, Z, U, X)$ vanishes. Plugging these two relations into (8) yields

$$
c\left(D_{U} D_{X} Y, Z\right)=(Z \wedge F(Y)-Y \wedge F(Z))(U, X)+F(U, X) c(Y, Z) .
$$

If $D$ is closed, (i.e. $F=0$ ) we thus get $D_{U} D_{X} Y=0$, which just means (by Lemma 3.3 again) that the vector field $D_{X} Y$ is the lift to $M$ of a vector field on $M_{1}$, and thus $D_{X}^{y} Y$ does not depend on $y$.

Conversely, if $D^{y}$ does not depend on $y$, we get $D_{U} D_{X} Y=0$, therefore $R^{D}(U, X) Y=0$. But the endomorphism $R^{D}(U, X): T M \rightarrow T M$ decomposes as a skew-symmetric endomorphism (in Riemannian geometry this is the only piece) and a symmetric one, which is equal to $F(U, X) \mathrm{Id}$. This piece has to vanish now that $R^{D}(U, X) Y=0$, hence $F(U, X)=0$. This, together with Lemma 4.6, proves that $F=0$.

REMARK 4.9. It follows from Lemma 4.6 that $D^{y}$ are closed Weyl structures on $M_{1}$, for any $y \in M_{2}$. Moreover, a $D^{y}$-parallel metric on $M_{1}$ is only determined up to a factor depending on $y$ alone, and the proof of Theorem 4.3 tells us to which proportionality class of sections of $L_{1} \rightarrow M_{1}$ it corresponds: Any section $\sigma_{2}$ of the scale bundle $L_{2} \rightarrow M_{2}$ induces a section $\tilde{\sigma}_{2}$ of $L \simeq p_{2}^{*} L_{2}$ over $M_{1} \times M_{2}$, which is, by definition, $D$-parallel in the directions of the $M_{1}$-leaves.

In other words, any metric $g_{2} \in c_{2}$ on $M_{2}$ defines a metric $g \in c$, such that the restriction of $g$ to the $M_{2}$ leaves is $g_{2}$ (leaf-independent metric), and $D^{y}$ is the Levi-Civita connection of the metric $\left.g\right|_{M_{1} \times\{y\}}$ (which depends on $y$ ). 
In Section 6.1 we will give more details about this construction.

REMARK 4.10. The results in this section hold under the implicit assumption that each factor of the conformal product has dimension at least one, because we need to identify the weight bundle of the product with the pull-back of those of each factor (see Remark 3.2).

5. Weyl-parallel forms. In this section we study the following problem, which motivates, as we shall see below, the notion of conformal product: Given a conformal manifold $\left(M^{n}, c\right)$, and a non-vanishing weightless $k$-form $\omega \in \Lambda_{0}^{k} M$ for some $1 \leq k \leq$ $n-1$, does there exist a Weyl structure $D$ such that $\omega$ is $D$-parallel?

REMARK 5.1. Since $\omega$ is not identically zero, a necessary condition for the existence of a Weyl structure $D$ with $D \omega=0$, is that $\omega$ is nowhere vanishing.

REMARK 5.2. We only consider weightless forms since if $T$ is a (non-vanishing) $D$ parallel weighted tensor, then $T / \sqrt{c(T, T)}$ is a $D$-parallel weightless tensor. Moreover, if $T$ has non-zero weight, the Weyl structure $D$ has to be exact (due to the fact that the conformal norm $c(T, T)$ is a $D$-parallel weight).

REMARK 5.3. For a conformal product $M=M_{1} \times M_{2}$, the weightless volume forms on the factors induce weightless forms which are parallel with respect to the unique adapted Weyl structure (which preserves the splitting $T M \simeq T M_{1} \oplus T M_{2}$ ). This will turn out to be one of the main examples of parallel weightless forms on conformal manifolds.

We start with the following useful result.

LEMmA 5.4. Let $\omega \in \Lambda_{0}^{k} M$ be a nowhere vanishing weightless $k$-form $(1 \leq k \leq$ $n-1)$. Then there exists at most one Weyl structure with respect to which $\omega$ is parallel.

Proof. Assume that $D \omega=D^{\prime} \omega=0$, denote $D^{\prime}=D+\tilde{\tau}$ like in (6) and let $g \in c$ be any ground metric, used to identify 1 -forms and vectors. For every vector field $X$ we get

$$
\left.\left.0=D_{X} \omega-D_{X}^{\prime} \omega=\tilde{\tau}_{X}(\omega)=X \wedge(\tau\lrcorner \omega\right)-\tau \wedge(X\lrcorner \omega\right)
$$

Taking the exterior product with $X$, and setting $X$ to be an element of some $g$ orthonormal basis $\left\{e_{i}\right\}$ we get, after adding up all the resulting equations,

$$
0=\tau \wedge \omega
$$

Similarly, taking the interior product with $X$ and summing over some $g$-orthonormal basis $X=e_{i}$ yields $\left.\left.0=(n-k+1)(\tau\lrcorner \omega\right)-\tau\right\lrcorner \omega$, thus $\left.\tau\right\lrcorner \omega=0$. This, together with (16) and the fact that $\omega$ is nowhere vanishing, implies $\tau=0$.

REMARK 5.5. Consider the linear map $\alpha: T^{*} M \rightarrow T^{*} M \otimes \Lambda_{0}^{k} M$ defined by

$$
\alpha(\tau)(X)=\tilde{\tau}_{X}(\omega)
$$

The proof of the lemma above show that if $\omega$ is a nowhere vanishing section of $\Lambda_{0}^{k} M$, then $\alpha$ is injective, and there exists a unique Weyl structure $D^{\omega}$ such that $D^{\omega} \omega$ is 
orthogonal to the image of $\alpha$. We call $D^{\omega}$ the minimal Weyl structure associated to $\omega$.

Our problem can thus be reformulated as follows: Given a conformal manifold $\left(M^{n}, c\right)$, find all nowhere vanishing sections $\omega$ of $\Lambda_{0}^{k} M$ for some $1 \leq k \leq n-1$, such that $D^{\omega} \omega=0$.

We start with the case where the minimal Weyl structure $D^{\omega}$ associated to $\omega$ is closed. Since our study is local, there exists some metric $g \in c$ whose Levi-Civita connection is $D^{\omega}$. Since $g$ trivializes the weight bundle, $\omega$ induces a parallel $k$-form on $M$. Using the Berger-Simons holonomy theorem, our problem in this case reduces to a purely algebraic one and its answer can be synthesized in the following classical statement.

TheOREM 5.6. Let $\left(M^{n}, g\right)$ be a simply connected Riemannian manifold with Levi-Civita covariant derivative $\nabla$. The space of parallel $k$-forms on $M$ is isomorphic to the space of fixed points of the holonomy group $\operatorname{Hol}(\nabla)$ of $\nabla$ acting on $\Lambda^{k}\left(\mathbb{R}^{n}\right)$. If $\operatorname{Hol}(\nabla)$ acts irreducibly on $\mathbb{R}^{n}$, then either $M=G / H$ is a symmetric space, $\operatorname{Hol}(\nabla)=$ $H$ and $H$ is listed in [2], Tables 1-4, pp.201-202, or $H o l(\nabla)$ belongs to the Berger list ([2], Corollary 10.92). If $\operatorname{Hol}(\nabla)$ acts reducibly on $\mathbb{R}^{n}$, then $\operatorname{Hol}(\nabla)$ is diagonally embedded in $\mathrm{SO}_{n}$ as a product $\mathrm{Hol}(\nabla)=H_{1} \times \ldots \times H_{l}$, where each $H_{i} \in \mathrm{SO}_{n_{i}}$ belongs to the lists above.

Another preliminary result concerns the special case of a weightless 2-form whose associated endomorphism is an almost complex structure $J$. This case is also classical and completely understood (see e.g. [11], Section 2):

Lemma 5.7. An almost complex structure $J$ compatible with the conformal structure on $\left(M^{2 m}, c\right)$ is parallel with respect to some Weyl structure $D$ if and only if

- $m=1$ or 2 and $J$ is integrable;

- $m \geq 3$ and $(M, c, J)$ is a locally conformally Kähler manifold.

Proof. We provide the proof for the reader's convenience. Assume that $D J=0$. Since $D$ is torsion free, $J$ is integrable ([8], Ch. 9, Corollary 3.5). Let $g$ be any metric in the conformal class $c$ with the associated 2-form $\omega(X, Y):=g(J X, Y)$. Using $g$, we identify 1 -forms with vectors, and 2-forms with skew-symmetric endomorphisms. If $\tau$ denotes the Lee form of $D^{J}$ with respect to the Levi-Civita connection $\nabla$ of $g$, we have for every tangent vector $X$

$$
0=D_{X}^{J}=\nabla_{X} J+\tilde{\tau}_{X} J
$$

From (5) we compute

$$
\begin{aligned}
\left(\tilde{\tau}_{X} J\right)(Y) & =\tau_{X}(J Y)-J\left(\tau_{X} Y\right) \\
& =\tau(X) J Y+\tau(J Y) X-g(X, J Y) \tau-J(\tau(X) Y+\tau(Y) X-c(X, Y) \tau) \\
& =(X \wedge J \tau+J X \wedge \tau)(Y)
\end{aligned}
$$

whence

$$
\nabla_{X} \omega=-(X \wedge J \tau+J X \wedge \tau)
$$

From (17) we get in a local orthonormal basis $\left\{e_{i}\right\}$ :

$$
d \omega=\sum_{i} e_{i} \wedge \nabla_{e_{i}} \omega=-\sum_{i} e_{i} \wedge\left(e_{i} \wedge \tau+J e_{i} \wedge \tau\right)=-2 \omega \wedge \tau .
$$


Taking the exterior derivative in this last relation yields

$$
0=d^{2} \omega=-2 d \omega \wedge \tau-2 \omega \wedge d \tau=-2 \omega \wedge d \tau
$$

For $m \geq 3$ the exterior product with $\omega$ is injective, so $d \tau=0$, and $(M, c, J)$ is thus locally conformally Kähler.

Conversely, assume first that $m \geq 3$ and $(M, c, J)$ is locally conformally Kähler. If $g_{1}$ and $g_{2}$ are local Kähler metrics on open sets $U_{1}$ and $U_{2}$ in the conformal class $c$, then $g_{1}$ and $g_{2}$ are homothetic on $U_{1} \cap U_{2}$. The Levi-Civita connections of all such metrics thus define a global Weyl structure $D$ leaving $J$ parallel.

If $m=2$ and $J$ is integrable, let $g$ be any metric in the conformal class $c$ with the associated 2-form $\omega(X, Y):=g(J X, Y)$. Since the wedge product with $\omega$ defines an isomorphism $\Lambda^{1} M \cong \Lambda^{3} M$, there exists a unique 1-form $\tau$ such that $d \omega=-2 \omega \wedge \tau$. From [8], Ch. 9, Proposition 4.2 we obtain

$$
\begin{aligned}
\nabla_{X} \omega(Y, Z) & =-(\omega \wedge \tau)(X, J Y, J Z)+(\omega \wedge \tau)(X, Y, Z) \\
& =-(J X \wedge \tau)(J Y, J Z)+(J X \wedge \tau)(Y, Z) \\
& =(X \wedge J \tau+J X \wedge \tau)(Y, Z)
\end{aligned}
$$

(notice that in the definition of $d \omega$ there is an extra factor 3 with the conventions in $[8])$. By (18), this means that $J$ is parallel with respect to the Weyl structure $\nabla+\tilde{\tau}$. $\square$

Before stating our main result, we need one more preliminary statement concerning conformal products.

Proposition 5.8. A conformal product $M=M_{1} \times M_{2}$, with $\operatorname{dim} M_{i}=n_{i} \geq 1$, admitting a non-trivial weightless form $\omega \in C^{\infty}\left(p_{1}^{*}\left(\Lambda_{0}^{k} M_{1}\right)\right), 1 \leq k \leq n_{1}-1$ which is parallel with respect to the adapted Weyl structure, is a closed conformal product.

In other words, on a non-closed conformal product, the only weightless forms of pure type $M_{1}$ or $M_{2}$ are the volume forms of the factors.

Proof. Let $M=M_{1} \times M_{2}$ and $T M=H_{1} \oplus H_{2}$ be the corresponding orthogonal splitting of the tangent space $H_{i} \simeq p_{i}^{*} T M_{i}$, where $p_{i}: M \rightarrow M_{i}$ are the canonical projections. Let $D$ be the adapted Weyl structure on $M$. Since $D_{X} \omega=0, \forall X \in H_{2}$, Lemma 3.3 shows that $\omega$ is the pull-back of a weightless form $\omega_{1} \in \mathbb{C}^{\infty}\left(\Lambda_{0}^{k} M_{1}\right)$, hence

$$
\omega=p_{1}^{*} \omega_{1}
$$

From Lemma 4.8,D induces a Weyl structure $D^{y}$ on each slice $M_{1} \times\{y\}$ and the equation $D_{Y} \omega=0, \forall Y \in H_{1}$, shows that the restriction of $\omega$ to each slice $M_{1} \times\{y\}$ is $D^{y}$-parallel. Of course, all these restrictions coincide with $\omega_{1}$, so we have

$$
D_{X}^{y} \omega_{1}=0, \forall X \in T M_{1} \text { and } \forall y \in M_{2} .
$$

Now, as the degree of $\omega_{1}$ lies between 1 and $n_{1}-1$, the equation above implies (using Lemma 5.4) that all Weyl structures $D^{y}$ coincide, so by Lemma 4.8 again, the Weyl structure $D$ is closed and $(M, c)$ is thus a closed conformal product.

We are now ready for the classification of conformal manifolds carrying conformally parallel forms.

THEOREM 5.9. Let $\left(M^{n}, c\right)$ be a conformal manifold and let $\omega \in \mathcal{C}^{\infty}\left(\Lambda_{0}^{k} M\right)$ be a weightless $k$-form $(1 \leq k \leq n-1)$ such that there exists a Weyl structure $D$ with respect to which $\omega$ is parallel. Then the following (non-exclusive) possibilities occur: 
1. D is closed, so Theorem 5.6 applies.

2. $M$ has dimension $4, k=2$, the endomorphism of $T M$ corresponding to $\omega$ is, up to a constant factor, an integrable complex structure, and D is its canonical Weyl structure.

3. $\left(M^{n}, c\right)$ is a conformal product of $\left(M^{n_{1}}, c_{1}\right)$ and $\left(M^{n_{2}}, c_{2}\right), D$ is the adapted Weyl structure, and

(a) if $n_{1} \neq n_{2}, \omega=\lambda \omega_{i}$ for some $\lambda \in \mathbb{R}$, where $\omega_{i}$ denotes the weightless volume form of the factor $M_{i}$;

(b) if $n_{1}=n_{2}$, then $\omega=\lambda \omega_{1}+\mu \omega_{2}$ for some $\lambda, \mu \in \mathbb{R}$.

Proof. Let us first consider the case of forms of low degree. If $\omega$ is a $D$-parallel weightless 1 -form, its kernel defines a $D$-parallel distribution of codimension 1 , so by Theorem $4.3(M, c)$ is a conformal product where one factor is one-dimensional and $\omega$ is its weightless volume form (case $3 a$ ).

The case when $\omega$ has degree 2 has a special geometrical meaning, since it can be seen as a skew-symmetric endomorphism $J$ of $T M$. As such, its square is a parallel symmetric endomorphism, therefore its eigenvalues are constant (and non-positive) and the corresponding eigenspaces are parallel. There are two cases to be considered.

If $J^{2}$ has only one eigenvalue, one may assume after rescaling that $J^{2}=-\operatorname{Id}_{T M}$, so by Lemma 5.7 , either $n=4$ and we are in case 2 , or $n \geq 6,(M, c)$ is locally conformally Kähler, and $D$ is closed (case 1 ).

If $J^{2}$ has at least two eigenvalues, we denote by $H_{1}$ one of the eigenspaces, and by $H_{2}$ its orthogonal complement in $T M$. The splitting $T M=H_{1} \oplus H_{2}$ is thus $D$-parallel, so $M$ has to be a non-trivial conformal product by Theorem 4.3. On the other hand, $J$ splits into $J=J_{1}+J_{2}$, where $J_{1}, J_{2}$ are the restrictions of $J$ to $H_{1}$, resp. $H_{2}$. The form $\omega$ splits accordingly into $\omega=\omega_{1}+\omega_{2}$, with $\omega_{i} \in \mathcal{C}^{\infty}\left(p_{i}^{*}\left(\Lambda_{0}^{2} M_{i}\right)\right)$. By Proposition 5.8, either the conformal product $M_{1} \times M_{2}$ is closed (case 1), or the non-trivial $\omega_{i}$ is a pull-back of a weightless volume form on $M_{i}$. Therefore, if $D$ is non-closed, $J^{2}$ has exactly two eigenvalues, which are either both non-zero (then $n=4$ and we are in case $3 \mathrm{~b}$ ) or only one is non-zero, and we are in case $3 \mathrm{a}$. Note that in the latter case, $\omega$ is defined as the pull-back of a volume form on a 2-dimensional conformal factor, thus it is decomposable.

In order to proceed, we make use of Merkulov-Schwachhöfer's classification of torsion-free connections with irreducible holonomy [9]. Their result, in the particular case of Weyl structures, states that there are four possibilities: Either $n=4$, or $D$ has full holonomy $\mathrm{CO}^{+}(n)$, or $D$ is closed, or $D$ has reducible holonomy.

If $n=4$, the case where the degree of $\omega$ is 1 or 2 has already been considered, and if $\omega$ has degree 3 , its Hodge dual is again a $D$-parallel weightless 1 -form, so we are in case $3 \mathrm{a}$.

If $D$ has full holonomy $\mathrm{CO}^{+}(n)$, there is of course no $D$-parallel weightless $k$-form on $M$ for $1 \leq k \leq n-1$.

If $D$ is closed we are already in case 1 .

For the rest of the proof, we thus may assume that the holonomy of $D$ acts reducibly on $T M$ and $\operatorname{dim} M \geq 5$. By Theorem $4.3,\left(M^{n}, c\right)$ is locally a conformal product of $\left(M^{n_{1}}, c_{1}\right)$ and $\left(M^{n_{2}}, c_{2}\right)$ and $D$ is the adapted Weyl structure. From Lemma 3.3 we have the following $D$-parallel decomposition:

$$
\Lambda_{0}^{k} M \simeq \bigoplus_{k_{1}+k_{2}=k} p_{1}^{*}\left(\Lambda_{0}^{k_{1}} M_{1}\right) \otimes p_{2}^{*}\left(\Lambda_{0}^{k_{2}} M_{2}\right)
$$

If for every $k_{1} \geq 1, k_{2} \geq 1$ the components of $\omega$ in $p_{1}^{*}\left(\Lambda_{0}^{k_{1}} M_{1}\right) \otimes p_{2}^{*}\left(\Lambda_{0}^{k_{2}} M_{2}\right)$ vanish, 
then $\omega=\omega_{1}+\omega_{2}$, with $\omega_{i} \in C^{\infty}\left(p_{i}^{*}\left(\Lambda_{0}^{k} M_{i}\right)\right)$, and $\omega_{1}, \omega_{2}$ are both parallel. Proposition 5.8 then implies that either $D$ is closed (case 1) or $\omega_{i}$ are both pull-backs of weightless volume forms on the factors (not both trivial). But this can only happen if $k$ is equal to one of the dimensions $n_{1}, n_{2}$ (case $3 \mathrm{a}$ ) or to both of them (case $3 \mathrm{~b}$ ).

To deal with the cases when $\omega$ is not a (combination of) pure type form, we may assume without loss of generality that $\omega$ is a non-trivial section of $p_{1}^{*}\left(\Lambda_{0}^{k_{1}} M_{1}\right) \otimes$ $p_{2}^{*}\left(\Lambda_{0}^{k_{2}} M_{2}\right)$ with $k_{1} \geq 1, k_{2} \geq 1$. By considering the Hodge dual of $\omega$ (which is $D$ parallel as well), we may even assume $k_{1}<n_{1}$ and $k_{2}<n_{2}$. We will show that, in this case, $D$ must be closed (case 1 ).

Let $R^{D}$ denote the curvature tensor of $D$. If $X \in T M_{1}$ and $A \in T M_{2}$, we have $R^{D}(\cdot, \cdot, X, A)=0$. Since $D \omega=0$ we also have $R^{D}(X, A)(\omega)=0$. From (8) we thus get

$$
R^{D}(X, A, \cdot, \cdot)=(F(X) \wedge A-F(A) \wedge X)(\cdot, \cdot)+F(X, A) c(\cdot, \cdot),
$$

and note that the first part is a skew-symmetric endomorphism of $T M$, and the second term is a multiple of the identity. That last one acts trivially on weightless forms, so we are left with four terms in $R^{D}(X, A)(\omega)$ and get:

$$
F(A) \wedge(X\lrcorner \omega)-X \wedge(F(A)\lrcorner \omega)-F(X) \wedge(A\lrcorner \omega)+A \wedge(F(X)\lrcorner \omega)=0,
$$

for all $X \in T M_{1}$ and $A \in T M_{2}$.

We now choose a metric $g \in c$ and identify the weightless form $\omega$ with the corresponding $p$-form of constant $g$-length. If $\theta$ denotes the Lee form of $D$ with respect to $g$ and $\nabla$ the Levi-Civita covariant derivative of $(M, g)$, we have

$$
\left.\left.\nabla_{U} \omega=-\tilde{\theta}_{U} \omega=\theta \wedge(U\lrcorner \omega\right)-U \wedge(\theta\lrcorner \omega\right) .
$$

By contraction we easily get $d \omega=-p \theta \wedge \omega$. Taking the exterior derivative in this relation yields

$$
d \theta \wedge \omega=0
$$

Since $d \theta=F$, taking the interior product with some vector in (22) yields

$$
F \wedge(U\lrcorner \omega)=-F(U) \wedge \omega, \quad \forall U \in T M .
$$

The rest of the proof is purely algebraic. Let $X_{i}, A_{j}$ be local orthonormal basis of $H_{1}:=T M_{1}$ and $H_{2}:=T M_{2}$. By Lemma 4.6 we have

$$
F=\sum_{i, j} f_{i j} X_{i} \wedge A_{j},
$$

therefore $F=\sum X_{i} \wedge F\left(X_{i}\right)=\sum A_{j} \wedge F\left(A_{j}\right)$. We also introduce the notations $\left.\left.\phi=\sum F\left(X_{i}\right) \wedge\left(X_{i}\right\lrcorner \omega\right)=-\sum A_{j} \wedge\left(F\left(A_{j}\right)\right\lrcorner \omega\right)$ and $\left.\psi=\sum F\left(A_{j}\right) \wedge\left(A_{j}\right\lrcorner \omega\right)=$ $\left.-\sum X_{i} \wedge\left(F\left(X_{i}\right)\right\lrcorner \omega\right)$.

For every $\alpha \in p_{1}^{*}\left(\Lambda_{0}^{p} M_{1}\right) \otimes p_{2}^{*}\left(\Lambda_{0}^{q} M_{2}\right)$ we have $\left.\sum X_{i} \wedge\left(X_{i}\right\lrcorner \alpha\right)=p \alpha$ and $\sum A_{j} \wedge$ $\left.\left(A_{j}\right\lrcorner \alpha\right)=q \alpha$. Taking the wedge product with $X$ in $(20)$, summing over $X=X_{i}$ and using (23) yields

$$
\left.0=-k_{1} F(A) \wedge \omega-F \wedge(A\lrcorner \omega\right)+A \wedge \psi=\left(1-k_{1}\right) F(A) \wedge \omega+A \wedge \psi .
$$


One last contraction with $A_{i}$ in (24) gives $\left(k_{1}+n_{2}-k_{2}\right) \psi=0$ (note that $\psi \in$ $\left.p_{1}^{*}\left(\Lambda_{0}^{k_{1}+1} M_{1}\right) \otimes p_{2}^{*}\left(\Lambda_{0}^{k_{2}-1} M_{2}\right)\right)$. Plugging back into (24) yields

$$
\left(1-k_{1}\right) F(A) \wedge \omega=0, \quad \forall A \in T M_{2} .
$$

In a similar way one obtains

$$
\left(1-k_{2}\right) F(X) \wedge \omega=0, \quad \forall X \in T M_{1},
$$

and, by replacing $\omega$ with its Hodge dual, and taking the Hodge dual of the equations for $* \omega$ analogous to $(25)$ and (26), we also get

$$
\begin{array}{ll}
\left.\left(n_{1}-k_{1}-1\right) F(A)\right\lrcorner \omega=0, & \forall A \in T M_{2}, \\
\left.\left(n_{2}-k_{2}-1\right) F(X)\right\lrcorner \omega=0, & \forall X \in T M_{1} .
\end{array}
$$

If $2 \leq k_{1} \leq n_{1}-2$, equations (25) and (27) show that $F=0$. Similarly, if $2 \leq k_{2} \leq n_{2}-2$, equations (26) and (28) show that $F=0$. We are thus left with four cases:

$$
\left(k_{1}, k_{2}\right) \in\left\{(1,1),\left(1, n_{2}-1\right),\left(n_{1}-1,1\right),\left(n_{1}-1, n_{2}-1\right)\right\} .
$$

Now, the pull-back to $M$ of the Hodge operator of $M_{1}$ defines an operator on $\Lambda_{0}^{*} M$, which maps each component $p_{1}^{*}\left(\Lambda_{0}^{k_{1}} M_{1}\right) \otimes p_{2}^{*}\left(\Lambda_{0}^{k_{2}} M_{2}\right)$ in the decomposition (19) isomorphically onto $p_{1}^{*}\left(\Lambda_{0}^{n_{1}-k_{1}} M_{1}\right) \otimes p_{2}^{*}\left(\Lambda_{0}^{k_{2}} M_{2}\right)$ by

$$
*_{1}\left[p_{1}^{*}\left(\omega_{1}\right) \wedge p_{2}^{*}\left(\omega_{2}\right)\right]:=p_{1}^{*}\left(* \omega_{1}\right) \wedge p_{2}^{*}\left(\omega_{2}\right) .
$$

One defines $*_{2}$ in a similar way and Lemma 3.3 shows that $*_{1}$ and $*_{2}$ are $D$-parallel. Using these "partial Hodge operators", it suffices to study only the first case in (29), i.e. we can assume that $\omega$ is a $D$-parallel 2 -form in $p_{1}^{*}\left(\Lambda_{0}^{1} M_{1}\right) \otimes p_{2}^{*}\left(\Lambda_{0}^{1} M_{2}\right)$.

As $n \geq 5$, by looking at the case of $D$-parallel 2-forms treated above, we see that $D$ is closed unless $\omega$ is decomposable. But this would imply that $\omega=\eta_{1} \wedge \eta_{2}$, where each of the weightless 1-forms $\eta_{i}$ define a $D$-parallel distribution included in $H_{i}$. The forms $\eta_{i}$ are thus $D$-parallel and Lemma 5.8 implies that $D$ is closed.

Looking back to the proof of Theorem 5.9, we see how different the non-closed case is from the case of a closed Weyl structure, and this despite the fact that the results are essentially similar: as in the classical Riemannian case, a weightless form which is parallel for a Weyl structure either defines a special irreducible holonomy, or the manifold is locally a product and the form is a linear combination of pull-backs of the volume forms of the factors (in the Riemannian case, other pull-backs may occur if the factors have reduced holonomy).

But while in Riemannian geometry the richer case is the one with irreducible, non-generic, holonomy - and these special geometries, despite extensive research in the last decades, are far from being completely understood -, in non-closed Weyl geometry this situation occurs only in dimension 4, and there it defines a rather simple structure. It appears that for non-closed Weyl structures it is the case with reduced holonomy which is more interesting, and the reason is that the holonomy group - although defining a local product structure on the manifold - is not itself a product like in the Riemannian situation.

The following consequence of the Theorem 5.9 sheds some light on the reduced holonomy group of a non-closed Weyl structure: 
Corollary 5.10. Let $\left(M_{1}^{n_{1}}, c_{1}\right)$ and $\left(M_{2}^{n_{2}}, c_{2}\right)$ be two conformal manifolds and let $c$ be a non-closed conformal product structure on $M=M_{1} \times M_{2}$ with adapted Weyl structure $D$. If the dimension $n$ is neither 2 nor 4 , then the only D-parallel distributions on $M$ are the kernels $H_{2}$ and $H_{1}$ of the canonical projections of $M$ on $M_{1}$, respectively $M_{2}$.

Proof. Let $\omega_{i}$ denote the (D-parallel) weightless volume form of $H_{i}$. If $H$ is a $D$-parallel distribution, its weightless volume form $\omega$ is $D$-parallel. Consider first the case $n=3$ : We can assume $\omega_{1}$ and $\omega$ are weightless 1-forms, and suppose they are not proportional (otherwise $H=H_{1}$ ). As the restriction of $\omega$ to $H_{1}$ is $D$-parallel as well, we can assume that there is a $D$-parallel orthogonal basis of 1 -forms on $M$. We denote by $L_{i}, i=1,2,3$, the three $D$-parallel, mutually orthogonal line distributions. By considering all three possible decompositions of $T M$ as $L_{i} \oplus L_{i}^{\perp}$, we see that Lemma 4.6 implies that $F=0$, hence $D$ is closed.

Suppose now that $D$ is non-closed and $\operatorname{dim}(M)>4$. The proof of Theorem 5.9 shows that $\omega$ is either proportional to $\omega_{1}$ or $\omega_{2}$ (in which case $H$ is equal to $H_{1}$ or $H_{2}$ ), or $n_{1}=n_{2}$ and $\omega=\lambda \omega_{1}+\mu \omega_{2}$ for some $\lambda, \mu \in \mathbb{R}^{*}$. We claim that this latter case is impossible. Let $X$ be some vector field in $H$ whose projections $X_{i}$ onto $H_{i}$ are both non-vanishing (such a vector field exists locally because $H$ is not equal to $H_{1}$ or $H_{2}$ ), and let $\sigma=c(X,$.$) be the dual 1-form of weight 1$, which decomposes correspondingly as $\sigma=\sigma_{1}+\sigma_{2}$. We clearly have $\sigma \wedge \omega=0$, whereas

$$
\sigma \wedge\left(\lambda \omega_{1}+\mu \omega_{2}\right)=\lambda \sigma_{2} \wedge \omega_{1}+\mu \sigma_{1} \wedge \omega_{2}
$$

and the two terms on the right hand side are non-vanishing and have bi-degree $\left(n_{1}, 1\right)$ and $\left(1, n_{2}\right)$ with respect to the decomposition (19). The assumption $n_{1}+n_{2} \neq 2$ shows that their sum can not vanish, a contradiction which proves our claim.

REMARK 5.11. The proposition above establishes a 1-1 correspondence between the non-closed Weyl structures $D$ on $M$ with reducible holonomy and conformal product structures, defined by a pair of complementary $D$-invariant distributions, provided $\operatorname{dim} M \neq 2,4$. Note that the restriction on the dimension of $M$ is necessary. Indeed, in dimension 2, any conformal product of two 1-dimensional manifolds admits parallel lines (distributions of rank 1) in any directions: Simply consider linear combinations of the two volume forms on the factors. But not all such conformal products are closed.

In dimension 4, there exist local examples of non-closed conformal multi-products, i.e., conformal manifolds admitting a non-closed Weyl structure that leaves invariant more than one pair of orthogonal distributions. These examples are described in Proposition 6.4 below.

\section{Examples and applications to Einstein-Weyl geometry.}

6.1. Curvature of a conformal product. Let $\left(M_{1}, g_{1}\right)$ and $\left(M_{2}, g_{2}\right)$ be Riemannian manifolds of dimensions $n_{1}$, resp. $n_{2}$, with $n:=n_{1}+n_{2}$, and let $f_{1}, f_{2}: M \rightarrow \mathbb{R}$ be $C^{\infty}$ functions, where $M:=M_{1} \times M_{2}$. Consider the following metric on $M$ :

$$
g:=e^{f_{1}} g_{1}+e^{f_{2}} g_{2}
$$

Then $(M,[g])$ is a conformal product of the conformal manifolds $\left(M_{1},\left[g_{1}\right]\right)$ and $\left(M_{2},\left[g_{2}\right]\right)$, in fact, any conformal product is locally of this form. Moreover, for two 
couples $\left(f_{1}, f_{2}\right)$, resp. $\left(f_{1}^{\prime}, f_{2}^{\prime}\right)$, the resulting conformal structures are equal if and only if $f_{1}-f_{2}=f_{1}^{\prime}-f_{2}^{\prime}$.

A conformal manifold $(M, c)$ is a closed conformal product if and only if $c$ has local representatives $g$ expressed in the form above, such that $f_{i}$ is the pull-back of a function on $M_{i}$ for $i=1,2$.

From Remark 4.9 (see also Lemma 3.3), the adapted Weyl structure $D$ of the conformal product $M=M_{1} \times M_{2}$ satisfies the following: The metric $e^{f_{1}-f_{2}} g_{1}+g_{2}$ is $D$-parallel in the $H_{1}$-directions, and $g_{1}+e^{f_{2}-f_{1}} g_{2}$ is $D$-parallel in the $H_{2}$-directions. This implies (in the sequel, $X_{i}, Y_{i}, Z_{i}$ are vector fields on $M_{i}$, equally considered as vector fields on the corresponding leaves on $M$ ):

1. $D_{X_{1}} X_{2}=D_{X_{2}} X_{1}=0$.

2. For $i=1,2, D_{X_{i}} Y_{i}$ coincides with the Levi-Civita connection of the metric $e^{\varepsilon(i)\left(f_{1}-f_{2}\right)} g_{i}$, where $\varepsilon(1):=1$ and $\varepsilon(2):=-1$.

3. the Lee form of $D$ with respect to $g$ is given by $\theta(X)=-X_{1}\left(f_{2}\right)-X_{2}\left(f_{1}\right)$, where $X_{i}$ denote the components of $X$ with respect to the decomposition $T M=T M_{1} \oplus T M_{2}$.

As before, we denote by $F$ the Faraday form of $D$, which by Lemma 4.6 satisfies

$$
F\left(X_{i}, Y_{i}\right)=0, \quad \forall X_{i}, Y_{i} \in T M_{i},
$$

so it is obtained by extending a section $F_{0}$ of $H_{1}^{*} \otimes H_{2}^{*}$ to a skew-symmetric bilinear form on $T M=H_{1} \oplus H_{2}$.

$F_{0}$ can also be extended to a symmetric bilinear form $\hat{F}$ on $T M$ :

$\hat{F}\left(X_{i}, Y_{i}\right):=0 ; \hat{F}\left(X_{1}, X_{2}\right):=F\left(X_{1}, X_{2}\right) ; \hat{F}\left(X_{2}, X_{1}\right):=-F\left(X_{2}, X_{1}\right), \forall X_{i}, Y_{i} \in T M_{i}$.

Lemma 6.1. Let $M:=M_{1} \times M_{2}$ be endowed with a conformal product structure $c:=\left[e^{f_{1}} g_{1}+e^{f_{2}} g_{2}\right]$. For $i=1,2$ denote by $\mathrm{Ric}^{i}$ the Ricci tensor of the metric $e^{\varepsilon(i)\left(f_{1}-f_{2}\right)} g_{i}$, viewed as a symmetric bilinear form on $T M_{i}$. Then the Ricci tensor of the adapted Weyl structure $D$ is given by

$$
\operatorname{Ric}^{D}=\operatorname{Ric}^{1} \oplus \operatorname{Ric}^{2}+\frac{2-n}{2} F+\frac{n_{1}-n_{2}}{2} \hat{F} .
$$

Proof. Recall that the Ricci tensor of a Weyl structure $D$ is defined by

$$
\operatorname{Ric}^{D}(X, Y):=\frac{1}{2} \sum_{k=1}^{n}\left(g\left(R_{X, e_{k}}^{D} e_{k}, Y\right)-g\left(R_{X, e_{k}}^{D} Y, e_{k}\right)\right)
$$

where $g$ is an arbitrary metric in the conformal class and $\left\{e_{k}\right\}$ is a local $g$-orthonormal frame (cf. [6], where, however, a different sign convention for the curvature tensor is used). It is straightforward to show that the skew-symmetric part of $\operatorname{Ric}^{D}$ equals $\frac{2-n}{2} F$ (this is actually an easy consequence of (8) and holds for any Weyl structure). If $X, Y$ are vector fields on $M_{1}$, each summand in (31) vanishes for $k \geq n_{1}+1$ since $R_{Z, T}^{D}$ preserves the splitting $T M=T M_{1} \oplus T M_{2}$ for all $Z, T$. Moreover, $R^{D}$ is just the Riemannian curvature of the metric $e^{f_{1}-f_{2}} g_{1}$ on vectors tangent to $M_{1}$. This shows that $\operatorname{Ric}^{D}(X, Y)=\operatorname{Ric}^{1}(X, Y)$ whenever $X$ and $Y$ are tangent to $M_{1}$ (and similarly $\operatorname{Ric}^{D}(X, Y)=\operatorname{Ric}^{2}(X, Y)$ for $X, Y$ tangent to $\left.M_{2}\right)$. Finally, using (8) we easily obtain $\operatorname{Ric}^{D}(X, Y)=\left(1-n_{2}\right) F(X, Y)$ and $\operatorname{Ric}^{D}(Y, X)=\left(1-n_{1}\right) F(Y, X)$ for $X \in T M_{1}$ and $Y \in T M_{2}$, which in turn implies (30). 
6.2. Einstein-Weyl conformal products. A Weyl manifold $(M, c, D)$ is called Einstein-Weyl if the trace-free symmetric part of the Ricci tensor $\operatorname{Ric}^{D}$ vanishes. In this subsection we will give the local characterization of all Einstein-Weyl structures $(M, c, D)$ in dimension 4 with reducible holonomy. Of course, we will be mainly interested in non-closed Weyl structures, the closed case being locally Riemannian, thus well-understood. Note that the scalar curvature of a non-closed Weyl structure, defined as usual as the trace of the Ricci tensor, is a section in a weight bundle and therefore never (covariantly) constant, unless it vanishes identically. The scalar-flat Einstein-Weyl structures are thus a special class of Weyl structures, which in our case provide examples of conformal products admitting multiple reductions (see previous section and Proposition 6.4).

Proposition 6.2. A non-closed Weyl manifold $(M, c, D)$ of dimension 4 , with reduced holonomy, is Einstein-Weyl if and only if it is locally isomorphic to a conformal product $M_{1} \times M_{2}, c=\left[g_{1}+e^{2 f} g_{2}\right]$, where $M_{1}$ and $M_{2}$ are open sets of $\mathbb{R}^{2}, g_{i}$ is the flat metric on $M_{i}$ and the function $f: M_{1} \times M_{2} \subset \mathbb{R}^{4} \rightarrow \mathbb{R}$ satisfies the Toda-type equation

$$
e^{2 f}\left(\partial_{11} f+\partial_{22} f\right)+\partial_{33} f+\partial_{44} f=0 .
$$

Moreover, the Einstein-Weyl structure is scalar flat if and only if $f$ is bi-harmonic (i.e. the restrictions of $f$ to $M_{1} \times\left\{x_{2}\right\}$ and to $\left\{x_{1}\right\} \times M_{2}$ are harmonic for all $x_{i} \in M_{i}$ ).

Proof. By Theorem 4.3, $(M, c, D)$ is reducible if and only if $(M, c)$ is a conformal product $M=M_{1}^{n_{1}} \times M_{2}^{n_{2}}, c=\left[g_{1}+e^{2 f} g_{2}\right]$, and $D$ is the adapted Weyl structure.

The first remark is that $M_{1}$ and $M_{2}$ must have the same dimension. Indeed, since the Faraday form is non-zero, Equation (30) shows that $(M, c, D)$ is Einstein-Weyl if and only if $n_{1}=n_{2}=2$ and

$$
\operatorname{Ric}^{1}+\operatorname{Ric}^{2}=\varphi\left(g_{1}+e^{2 f} g_{2}\right)
$$

for some function $\varphi: M \rightarrow \mathbb{R}$, where we remind that $\operatorname{Ric}^{i}$ is the Ricci tensor of the metric $e^{2 \varepsilon(i) f} g_{i}$. Every 2-dimensional metric being locally conformal to the flat metric, one can assume that $g_{1}$ and $g_{2}$ are flat. Using the basic formulas for the conformal change of the Ricci tensor ([2], 1.159d), (33) becomes

$$
-\left(\Delta_{1} f\right) g_{1}+\left(\Delta_{2} f\right) g_{2}=\varphi\left(g_{1}+e^{2 f} g_{2}\right)
$$

where $\Delta_{i}$ denote the partial Laplacians on $\mathbb{R}^{4}=\mathbb{R}^{2} \times \mathbb{R}^{2}$ :

$$
\Delta_{1} f:=\partial_{11} f+\partial_{22} f, \quad \Delta_{2} f:=\partial_{33} f+\partial_{44} f .
$$

Equation (34) is clearly equivalent to (32). Moreover, the trace of $\operatorname{Ric}^{D}$ with respect to the metric $g_{1}+e^{2 f} g_{2}$ is $-2 \Delta_{1} f+2 e^{-2 f} \Delta_{2} f$. This shows that $D$ is Einstein-Weyl and scalar-flat if and only if $\Delta_{1} f=\Delta_{2} f=0$.

REMARK 6.3. If $f: U \times V \rightarrow \mathbb{R}, U, V \subset \mathbb{C}$ is bi-harmonic, i.e., harmonic with respect to both variables $z \in U, w \in V$, then

$$
f=\operatorname{Re}(F)+\operatorname{Re}(\tilde{G}),
$$

where $F: U \times V \rightarrow \mathbb{C}$ and $G: U \times \bar{V} \rightarrow \mathbb{C}$ are holomorphic, and $\tilde{G}(z, w):=G(z, \bar{w})$, $\forall(z, w) \in U \times V$. It turns out that the case where one of $F, G$ is trivial can be characterized geometrically: 
Proposition 6.4. A non-closed conformal product $(M, c, D)$ (where $M:=U_{1} \times$ $U_{2}, U_{1}, U_{2} \subset \mathbb{R}^{2}, c:=\left[g_{1}+e^{2 f} g_{2}\right]$, and $g_{i}$ is the Euclidean metric on $\left.U_{i}\right)$ is hyperHermitian if and only if $f$ is the real part of a holomorphic function in the complex variables $z:=x_{1} \pm i x_{2} \in U_{1} \subset \mathbb{R}^{2}$ and $w:=x_{3} \pm i x_{4} \in U_{2}$.

Proof. If $f$ is the real part of a holomorphic function $h(z, w)$, then the metric reads $g=g_{1}+\left|e^{h}\right|^{2} g_{2}$ and is thus hyper-Hermitian by [10], $\S 7$.

Conversely, assume that $\left(M, c:=\left[g_{1}+e^{2 f} g_{2}\right], D\right)$ is hyper-Hermitian. If $I_{1}$, $I_{2}$ denote the $D$-parallel weightless volume forms of the factors, viewed as skewsymmetric endomorphisms on $M$, then $\pm I_{1}, \pm I_{2}$ are $D$-parallel Hermitian structures on $M$. Up to a choice of signs of $I_{1}, I_{2}$ (choice that determines the signs in the definition of the complex variables $z, w)$, one might assume that $I:=I_{1}+I_{2}$ belongs to the hyper-Hermitian structure, i.e., for any unitary vectors $Y_{i} \in V_{i}$, the orthonormal frame $Y_{1}, I_{1} Y_{1}, Y_{2}, I_{2} Y_{2}$ is positively oriented.

Let $J$ be another integrable Hermitian structure anti-commuting with $I$. We denote by $X_{i}:=\partial / \partial x_{i}$ for $1 \leq i \leq 4$, so $I X_{1}=X_{2}$ and $I X_{3}=X_{4}$. Then one can write $J X_{1}=a X_{3}+b X_{4}$ for some functions $a, b: M \rightarrow \mathbb{R}$. Since $J$ is Hermitian we must have $e^{2 f}\left(a^{2}+b^{2}\right)=1$ and the anti-commutation with $I$ yields $J X_{2}=b X_{3}-a X_{4}$. A straightforward computation using the vanishing of the Nijenhuis tensor of $J$ yields

$$
\begin{aligned}
0=N^{J}\left(X_{1}, X_{2}\right)= & \left(\partial_{1} b-\partial_{2} a\right) J\left(X_{3}\right)-\left(\partial_{1} a+\partial_{2} b\right) J\left(X_{4}\right) \\
& +\left(\partial_{4} a+\partial_{3} b\right)\left(a X_{3}-b X_{4}\right)-\left(\partial_{3} a-\partial_{4} b\right)\left(b X_{3}+a X_{4}\right) .
\end{aligned}
$$

This is of course equivalent to the fact that $H:=a+i b$ is holomorphic in the complex variables $z:=x_{1}-i x_{2}$ and $w:=x_{3}+i x_{4}$, so $f=-\ln |H|=\operatorname{Re}(-\ln H)$ is the real part of a holomorphic function.

This proposition has a few consequences:

1. The examples of 4-dimensional hyper-Hermitian manifolds constructed by Joyce (cf. [7], or [10], §7) are actually conformal products.

2. In contrast with the Riemannian case, where a Ricci-flat Kähler surface is automatically hyper-Kähler, there exist examples of scalar-flat Einstein-Weyl Hermitian manifolds in dimension 4 which are not hyper-Hermitian. Indeed, it suffices to choose $f$ bi-harmonic, but not the real part of a holomorphic function in any of the variables $z:=x_{1} \pm i x_{2}$ and $w:=x_{3} \pm i x_{4}$, e.g. $f(z, w)=$ $\operatorname{Re}(z) \operatorname{Re}(w)=x_{1} x_{3}$. On the other hand the complex structure on $M$ defined by the complex structures of the factors is clearly $D$-parallel.

3 . The hyper-Hermitian non-closed conformal products are exactly the nonclosed conformal multi-products, i.e., whose adapted Weyl structure $D$ leaves more than one pair of complementary distributions. The restricted holonomy of these special structures is $\mathbb{C}^{*} \subset \mathrm{CO}(4)$, as the following proposition states:

Proposition 6.5. A 4-dimensional non-closed conformal product $(M, c, D)$ that admits multiple D-parallel splittings is hyper-Hermitian, and is locally isomorphic to one of the examples described in Proposition 6.4. The holonomy of $D$ is then $\mathbb{C}^{*}$, acting by scalar multiplication on $\mathbb{C}^{2} \simeq T M$.

Proof. A parallel splitting corresponds to a pair of $D$-parallel decomposable weightless forms. If two such splittings exist, the factors $M_{1}, M_{2}$ of $M$ need to have the same dimension (Theorem 5.9). Denoting, as before, by $I_{1}$ and $I_{2}$ the weightless volume forms of the 2-dimensional factors $M_{1}$ and $M_{2}$ respectively, we have a 2-dimensional space of $D$-parallel weightless forms, but the only splitting that they 
define is the original one. Therefore, there must be another $D$-parallel 2-form $\omega$, orthogonal to both $I_{1}$ and $I_{2}$.

Viewing $\omega$ as a skew-symmetric endomorphism, we can distinguish two cases: Either its square has only one eigenvalue, thus $\omega$ defines a $D$-parallel complex structure $J$, orthogonal to $I_{1}$ and $I_{2}$, or $\omega$ defines itself an orthogonal splitting, in which case, the sum of the two weightless volume forms of those factors defines a $D$-parallel complex structure $J$ as well.

By an appropriate change of signs of the weightless volume forms $I_{1}, I_{2}$, we can assume that $I:=I_{1}+I_{2}$ defines the same orientation as $J$. With respect to this orientation of $M$, the 2 -forms $I$ and $J$ are self-dual and orthogonal, thus they anti-commute as endomorphisms and define therefore a $D$-parallel hyper-Hermitian structure on $M$. Proposition 6.4 applies and we have a local model. We shall see below that the Faraday form $F$ is, in this case, anti-self-dual.

For the second claim, we first note that the holonomy representation of $D$ preserves all self-dual forms and at least one anti-self-dual 2-form, namely $\tilde{I}:=I_{1}-I_{2}$, thus $\operatorname{Hol}_{0}(D) \subset \mathbb{C}^{*}$.

In order to prove that the restricted holonomy of $D$ is exactly $\mathbb{C}^{*}$, we will show that the Lie algebra of $\operatorname{Hol}_{0}(D)$ is 2-dimensional.

First, denote as before by $X_{i}, Y_{i}$ arbitrary vector fields on $M_{i}$, lifted to vector fields on $M$. We have

$$
R_{X_{1}, Y_{1}}^{D} X_{2}=0 ; R_{X_{2}, Y_{2}}^{D} X_{1}=0,
$$

and, since $\Delta_{i} f=0$, the metric $e^{ \pm 2 f} g_{i}$ is flat, therefore

$$
R_{X_{i}, Y_{i}}^{D} Z_{i}=0
$$

hence

$$
R^{D}\left(I_{i}^{\sharp}\right)=0 .
$$

Here we view the curvature operator $R^{D}$ as being defined on $\Lambda^{2}(T M)$ with values in $\operatorname{End}(T M)$. We will show that the image of $R^{D}$ is 2-dimensional in every point where $F$ does not vanish.

We have used the following notation: $\sharp: T^{*} M \rightarrow T M$ and its inverse $b: T M \rightarrow$ $T^{*} M$ are the canonical isomorphisms for a particular choice of a metric in the conformal class (fixed once and for all), traditionally called "raising", respectively, "lowering" of indices. The index $\sharp$, respectively $b$, attached once to a tensor, signifies the raising, respectively lowering of one index. For example, $F^{\sharp}$ is a skew-symmetric endomorphism of $T M$, and $F^{\sharp \sharp}$ is a bi-vector, i.e. a section in $\Lambda^{2}(T M)$.

Straightforward computations show:

$$
\begin{aligned}
R_{X_{1}, X_{2}}^{D} Y & =F\left(X_{1}, X_{2}\right) Y+(F \wedge \mathrm{Id})\left(X_{1}, X_{2}\right)(Y) \\
& =F\left(X_{1}, X_{2}\right) Y+\left[F^{\sharp}, X_{1} \wedge X_{2}^{b}\right](Y),
\end{aligned}
$$

where the bracket $[\cdot, \cdot]$ denotes the commutator in $\operatorname{End}(T M)$.

We infer that the skew-symmetric endomorphism $\left[F^{\sharp}, X_{1} \wedge X_{2}^{b}\right]$ is always antiself-dual (since it commutes with the basis of the space of self-dual endomorphisms, defined by the $D$-parallel hyper-Hermitian structure), which implies that $F$ itself is anti-self-dual. 
Another consequence of (35) is that

$$
R^{D}(\alpha)=\langle F, \alpha\rangle \mathrm{Id}+\left[F^{\sharp}, \alpha^{b}\right],
$$

for any 2 -vector $\alpha$ orthogonal on both $I_{i}^{\sharp \sharp}, i=1,2$. In particular, in a point where $F \neq 0$,

$$
R^{D}(F)=\|F\|^{2} \operatorname{Id} \text { and } R^{D}(\beta)=\left[F^{\sharp}, \beta^{b}\right]
$$

where $\tilde{I}^{b}, F, \beta^{b b}$ is an orthogonal basis of $\Lambda^{-} M$.

Thus $R^{D}(F)$ and $R^{D}(\beta)$ are linearly independent at any point where $F \neq 0$, since the first is a multiple of the identity and the second is a skew-symmetric endomorphism. That means that the image through the curvature operator of $\Lambda^{2}(T M)$ is 2-dimensional on an open set, hence the holonomy algebra is 2-dimensional, more precisely $\operatorname{Hol}_{0}(D)=\mathbb{C}^{*}$ as claimed.

\section{REFERENCES}

[1] S. Armstrong, Definite signature conformal holonomy: A complete classification, J. Geom. Phys., 57 (2007), pp. 2024-2048.

[2] A. Besse, Einstein manifolds, Ergebnisse der Mathematik und ihrer Grenzgebiete (3) 10. Springer-Verlag, Berlin, (1987).

[3] D.M.J. Calderbank, Selfdual Einstein metrics and conformal submersions, arXiv:math/0001041v1 (2000).

[4] D.M.J. Calderbank, The geometry of the Toda equation, J. Geom. Phys., 36 (2000), pp. 152162 .

[5] J.C. González-Dávila, F. Martin Cabrera, M. Salvai, Harmonicity of sections of sphere bundles, Math. Z., 261:2 (2009), pp. 409-430.

[6] P. Gauduchon, Structures de Weyl-Einstein, espaces de twisteurs et variétés de type $S^{1} \times S^{3}$, J. reine angew. Math., 469 (1995), pp. 1-50.

[7] D. Joyce, Explicit Construction of Self-dual 4-Manifolds, Duke Math. J., 77:3 (1995), pp. 519 552.

[8] S. Kobayashi, K. Nomizu, Foundations of Differential Geometry II, New York, Interscience Publishers, 1969.

[9] S. Merkulov, L. SchwachHöFER, Classification of irreducible holonomies of torsion-free affine connections, Annals of Mathematics, 150:1 (1999), pp. 77-149.

[10] A. Moroianu, Structures de Weyl admettant des spineurs parallèles, Bull. Soc. Math. France, 124 (1996), pp. 685-695.

[11] H. Pedersen, Y. S. Poon, A. Swann, The Einstein-Weyl equations in complex and quaternionic geometry, Diff. Geom. Appl., 3 (1993), pp. 309-321. 
F. BELGUN AND A. MOROIANU 